\title{
Heteronuclear fermionic superfluids with spin degrees of freedom
}

\author{
D. B. M. Dickerscheid ${ }^{1}$ 田 Y. Kawaguchi ${ }^{1}$, and M. Ueda ${ }^{1,2}$ \\ ${ }^{1}$ Department of Physics, Tokyo Institute of Technology, \\ 2-12-1 Ookayama, Meguro-ku, Tokyo 152-8551, Japan and \\ ${ }^{2}$ ERATO, Macroscopic Quantum Control Project, JST, Bunkyo-ku, Tokyo 113-8656, Japan
}

(Dated: December 1, 2018)

\begin{abstract}
We present a theory of spinor superfluidity in a two-species heteronuclear ultracold fermionic atomic gas consisting of arbitrary half-integer spin and one-half spin atoms. In particular, we focus on the magnetism of the superfluid phase and determine the possible phases in the absence of a magnetic field. Our work demonstrates similarities between heteronuclear fermionic superfluids and spinor Bose-Einstein condensates at the mean-field level. Possible experimental situations are discussed.
\end{abstract}

PACS numbers: 03.75.Fi, 67.40.-w, 32.80.Pj, 39.25+k

\section{INTRODUCTION.}

Fermionic superfluidity is currently one of the most active research topics in the field of ultracold atomic gases. Fermionic superfluids have been realized and the BECBCS crossover regime has been explored for both balanced and imbalanced systems [1, 2, 3, 4, 4, ㅎ, 6, 7, 8, 9]. Another line of research that has attracted a growing interest concerns mixtures of different atomic species 10, 11, 12, 13, 14 such as two-species Fermi-Fermi mixtures. Fermionic heteronuclear gases have currently been investigated both experimentally [15] and theoretically [16, 17, 18, 19]. Recently, the first quantum degenerate two-species Fermi-Fermi mixture has been realized [20].

In this paper we theoretically investigate superfluid properties of a heteronuclear Fermi-Fermi mixture with spin degrees of freedom. Two-species Fermi mixtures differ from the single-species gas in such a fundamental way that at low temperatures atoms in the same spin state can interact with each other through $s$-wave scattering. For identical fermions, however, this is prohibited due to the Pauli principle and consequently superfluidity of a single species of atoms occurs in the spin-singlet state for the $s$ wave channel. Specifically, for the case where both atoms have spin one-half this means that both spin-singlet and spin-triplet states are allowed. Theoretical investigations of superfluidity of heteronuclear Fermi gases have so far not explored the physics of the (hyperfine) spin degrees of freedom of the atoms [18]. While pairing in single-species fermionic superfluids with arbitrary spin has been studied 21, 22], no attempt to investigate the combined effects of those two, i.e., heteronuclear spinor superfluidity, has been made to the best of our knowledge. This is the subject we address in this work. In particular, our work demonstrates a strong similarity between heteronuclear superfluids and spinor BECs at the mean-field level and suggests the existence of novel many-body states.

Experimentally, a particularly interesting candidate

*Electronic address: dd1978@gmail.com to realize a heteronuclear spinor superfluid is an isotope mixture of the rare-earth element ytterbium $(\mathrm{Yb})$, which has two stable fermionic isotopes with nuclear spin $I=1 / 2\left({ }^{171} \mathrm{Yb}\right)$ and $I=5 / 2\left({ }^{173} \mathrm{Yb}\right)$, respectively, and electronic spin $S=0$. Both isotopes have already been trapped optically and recently the ${ }^{173} \mathrm{Yb}$ gas was cooled to quantum degeneracy [23].

This paper is organized as follows. We formulate our problem in Sec. IIA and develop the Bogoliubov and Ginzburg-Landau theories for our system in Secs. IIB and IIC respectively. We apply the theory for specific cases in Sec. [III and discuss possible experimental realization of the gases considered here in Sec. IV]. We summarize and conclude this paper in Sec. V. Some algebraic manipulations to derive the Bogoliubov excitation spectrum of a heteronuclear superfluid have been relegated to the Appendix to avoid disgressing from the main subject.

\section{THEORY OF HETERONUCLEAR FERMIONIC SUPERFLUIDITY}

\section{A. Formulation of the problem}

We consider an optically trapped Fermi-Fermi mixture with spins $f_{\phi} \geq 1 / 2$ and $f_{\chi}=1 / 2$, where we distinguish the two atomic species with suffixes $\phi$ and $\chi$. We assume that the temperature is sufficiently low so that we have only to consider $s$-wave interactions between the atoms. In general, there are interspecies and intraspecies interactions, and experimentally it is possible to tune the interspecies interactions independently of the intraspecies interactions. In this paper we will ignore the intraspecies interactions to focus on the bare essentials of this system. The total spin $f$ of the interacting atoms with spin $f_{\phi} \geq 1 / 2$ and $f_{\chi}=1 / 2$ is given by $f^{ \pm} \equiv f_{\phi} \pm 1 / 2$. In the absence of an external magnetic field, the total Hamiltonian $\hat{H}=\hat{H}_{1 B}+\hat{V}$ is the sum of one-body part $\hat{H}_{1 B}$ and interaction $\hat{V}$. The general form of the interaction can 
be represented as

$$
\hat{V}=\sum_{f=f^{ \pm}} V_{f}\left(\boldsymbol{x}-\boldsymbol{x}^{\prime}\right) \hat{\mathcal{P}}_{f}
$$

where the projection operators $\hat{\mathcal{P}}_{f}$ project pairs of atoms onto pairs of the total spin $f$ channel and $V_{f}\left(\boldsymbol{x}-\boldsymbol{x}^{\prime}\right)$ is the interaction potential between a pair of atoms with total spin $f$. For $s$-wave interactions, the interaction potential can be approximated by a pseudopotential $V_{f}\left(\boldsymbol{x}-\boldsymbol{x}^{\prime}\right)=$ $g_{f} \delta\left(\boldsymbol{x}-\boldsymbol{x}^{\prime}\right)$.

In second quantization the one-body Hamiltonian is given by

$$
\begin{aligned}
\hat{H}_{1 B} & =\int d \boldsymbol{x} \sum_{\sigma=-f_{\phi}}^{f_{\phi}} \hat{\phi}_{\sigma}^{\dagger}(\boldsymbol{x})\left(-\frac{\hbar^{2} \nabla^{2}}{2 M_{\phi}}+V_{\phi, \sigma}^{\mathrm{ex}}(\mathbf{x})-\mu_{\phi}\right) \hat{\phi}_{\sigma}(\boldsymbol{x}) \\
& +\int d \boldsymbol{x} \sum_{\sigma= \pm 1 / 2} \hat{\chi}_{\sigma}^{\dagger}(\boldsymbol{x})\left(-\frac{\hbar^{2} \nabla^{2}}{2 M_{\chi}}+V_{\chi, \sigma}^{\mathrm{ex}}(\boldsymbol{x})-\mu_{\chi}\right) \hat{\chi}_{\sigma}(\boldsymbol{x})
\end{aligned}
$$

where $\hat{\phi}_{\sigma}^{\dagger}\left(\hat{\phi}_{\sigma}\right)$ and $\hat{\chi}_{\sigma}^{\dagger}\left(\hat{\chi}_{\sigma}\right)$ are the creation (annihilation) operators of atoms of spin $f_{\phi}$ and spin $1 / 2$, respectively, in the magnetic sublevel $\sigma$.

The terms $V_{\phi, \sigma}^{\mathrm{ex}}(\boldsymbol{x})$ and $V_{\chi, \sigma}^{\mathrm{ex}}(\boldsymbol{x})$ describe statedependent external potentials for the $\phi$ and $\chi$ atoms, respectively, $\mu_{\phi}$ and $\mu_{\chi}$ are the chemical potentials, and $M_{\phi}$ and $M_{\chi}$ are the masses of the $\phi$ and $\chi$ atoms, respectively. The projection operators can be expressed in second quantisation as

$$
\hat{\mathcal{P}}_{f}=\sum_{m} \hat{A}_{f, m}^{\dagger}(\boldsymbol{x}) \hat{A}_{f, m}(\boldsymbol{x})
$$

where

$$
\hat{A}_{f, m}(\boldsymbol{x})=\sum_{\sigma, \sigma^{\prime}}\left\langle f, m \mid f_{\phi}, \sigma ; 1 / 2, \sigma^{\prime}\right\rangle \hat{\phi}_{\sigma}(\boldsymbol{x}) \hat{\chi}_{\sigma^{\prime}}(\boldsymbol{x})
$$

is the annihilation operator of a pair of $\phi$ and $\chi$ atoms with total spin $f$ and total magnetic quantum number $m$, with $\left\langle f, m \mid f_{\phi}, \sigma ; 1 / 2, \sigma^{\prime}\right\rangle$ being a Clebsch-Gordan coefficient. The interaction Hamiltonian is then given by

$$
\hat{V}=\frac{1}{2} \sum_{f, m} \int d \boldsymbol{x} g_{f} \hat{A}_{f, m}^{\dagger}(\boldsymbol{x}) \hat{A}_{f, m}(\boldsymbol{x}) .
$$

Since we are interested in the superfluid phase we assume the interaction between the atoms to be attractive, i.e., $g_{f}<0$.

The interaction Hamiltonian in Eq. (1) can be rewritten in a physically suggestive manner. It follows from the completeness relation $1=\hat{\mathcal{P}}_{f+}+\hat{\mathcal{P}}_{f-}$ and from the total spin squared

$$
\left(\boldsymbol{f}_{\chi}+\boldsymbol{f}_{\phi}\right)^{2}=\sum_{f=f^{+}, f^{-}} f(f+1) \hat{\mathcal{P}}_{f}
$$

with $f^{ \pm} \equiv f_{\phi} \pm 1 / 2$ that

$$
\hat{\mathcal{P}}_{f^{ \pm}}=\frac{1}{2 f^{+}}\left[f^{+} \pm \frac{1}{2} \pm 2 \boldsymbol{f}_{\chi} \cdot \boldsymbol{f}_{\phi}\right]
$$

The interaction Hamiltonian can then be constructed as

$$
\begin{aligned}
\hat{V}= & \left(g_{f^{+}} \hat{\mathcal{P}}_{f^{+}}+g_{f^{-}} \hat{\mathcal{P}}_{f^{-}}\right) \delta\left(\boldsymbol{x}-\boldsymbol{x}^{\prime}\right) \\
= & \left\{g_{f^{+}} \frac{f^{+}+\frac{1}{2}}{2 f^{+}}+g_{f^{-}} \frac{f^{+}-\frac{1}{2}}{2 f^{+}}\right\} \delta\left(\boldsymbol{x}-\boldsymbol{x}^{\prime}\right) \\
& +\frac{\left(g_{f^{+}}-g_{f^{-}}\right)}{f^{+}} \delta\left(\boldsymbol{x}-\boldsymbol{x}^{\prime}\right) \boldsymbol{f}_{\chi} \cdot \boldsymbol{f}_{\phi} .
\end{aligned}
$$

The corresponding second-quantized expression is

$$
\begin{aligned}
& \hat{V}=\frac{1}{2} \int d \boldsymbol{x} \frac{\left(g_{f^{+}}-g_{f^{-}}\right)}{f^{+}}:{ }^{\chi} \hat{\boldsymbol{F}}(\boldsymbol{x}) \cdot{ }^{\phi} \hat{\boldsymbol{F}}(\boldsymbol{x}): \\
& +\frac{1}{2} \int d \boldsymbol{x}\left\{g_{f^{+}} \frac{f^{+}+\frac{1}{2}}{2 f^{+}}+g_{f^{-}} \frac{f^{+}-\frac{1}{2}}{2 f^{+}}\right\}: \hat{n}_{\chi}(\boldsymbol{x}) \hat{n}_{\phi}(\boldsymbol{x}):
\end{aligned}
$$

where :: denotes normal ordering, $\hat{n}_{\phi}(\boldsymbol{x})$ and $\hat{n}_{\chi}(\boldsymbol{x})$ are the total densities of the $\phi$ and $\chi$ atoms given by

$$
\hat{n}_{\phi}(\boldsymbol{x})=\sum_{\sigma} \hat{\phi}_{\sigma}^{\dagger}(\boldsymbol{x}) \hat{\phi}_{\sigma}(\boldsymbol{x})
$$

and

$$
\hat{n}_{\chi}(\boldsymbol{x})=\sum_{\sigma} \hat{\chi}_{\sigma}^{\dagger}(\boldsymbol{x}) \hat{\chi}_{\sigma}(\boldsymbol{x})
$$

and $\chi \hat{\boldsymbol{F}}(\boldsymbol{x})$ and ${ }^{\phi} \hat{\boldsymbol{F}}(\boldsymbol{x})$ denote the spin density vectors whose components are given by

$$
{ }^{\phi} \hat{F}^{i}(\boldsymbol{x})=\sum_{\sigma, \sigma^{\prime}} \hat{\phi}_{\sigma}^{\dagger}(\boldsymbol{x})\left[{ }^{\phi} F^{i}\right]_{\sigma \sigma^{\prime}} \hat{\phi}_{\sigma^{\prime}}(\boldsymbol{x})
$$

and

$$
{ }^{\chi} \hat{F}^{i}(\boldsymbol{x})=\sum_{\sigma, \sigma^{\prime}} \hat{\chi}_{\sigma}^{\dagger}(\boldsymbol{x})\left[{ }^{\chi} F^{i}\right]_{\sigma \sigma^{\prime}} \hat{\chi}_{\sigma^{\prime}}(\boldsymbol{x}),
$$

respectively, where $\left[{ }^{\phi} F^{i}\right]_{\sigma \sigma^{\prime}}$ and $\left[{ }^{\chi} F^{i}\right]_{\sigma \sigma^{\prime}}$ are the matrix elements of the $i=x, y, z$ components of spin matrix vectors ${ }^{\phi} \boldsymbol{F}$ and $\chi_{\boldsymbol{F}}$. The sign of $\left(g_{f^{+}}-g_{f^{-}}\right)$specifies which of the two spin states $f^{+}$and $f^{-}$is energetically favorable and thus determines if the total angular momentum of the Cooper pairs is $f^{+}$or $f^{-}$. The generic phase diagram that results is sketched in Fig. 11 At high temperatures, the system is in the normal state and the total angular momentum is not fixed. When we lower the temperature for a fixed value of $g_{f^{-}}$and $\left|g_{f^{+}}\right|>\left|g_{f^{-}}\right|$, we expect a phase transition to a BCS superfluid whose total angular momentum of each Cooper pair is $f^{+}$and the transition temperature is determined by $g_{f}$. On the other hand, when $\left|g_{f^{+}}\right|<\left|g_{f^{-}}\right|$, we expect a BCS superfluid with total angular momentum $f^{-}$. By changing the ratio $g_{f} / g_{f^{-}}$we can switch between the two types of superfluidity. 


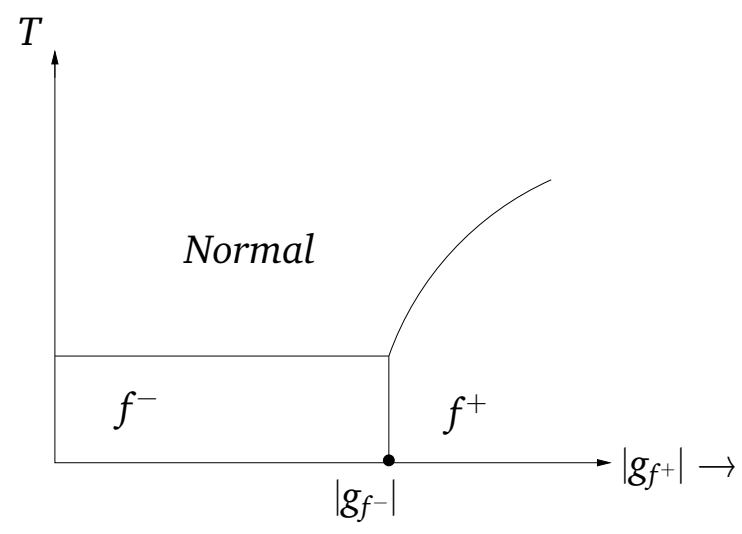

FIG. 1: Generic phase diagram as a function of temperature $T$ and coupling constant $g_{f^{+}} \cdot f^{-}\left(f^{+}\right)$denotes the phase in which the spin angular momentum of a constituent Cooper pair is $f^{-}\left(f^{+}\right)$.

\section{B. Mean-field theory of heteronuclear superfluidity}

The phases we found in the previous subsection possess the internal spin degrees of freedom. To investigate the magnetic properties of the superfluid phases we make use of mean-field theory. The order parameter is defined by

$$
\Delta_{f, m}(\boldsymbol{x}) \equiv g_{f}\left\langle\hat{A}_{f, m}(\boldsymbol{x})\right\rangle \text {. }
$$

Applying the Gorkov decoupling, we decompose the interaction Hamiltonian as

$\hat{V}=\frac{1}{2} \int d \boldsymbol{x} \sum_{m}\left(\hat{A}_{f, m}^{\dagger}(\boldsymbol{x}) \Delta_{f, m}+\Delta_{f, m}^{*} \hat{A}_{f, m}-\frac{\left|\Delta_{f, m}\right|^{2}}{g_{f}}\right)$.

In a homogeneous system, the resulting mean-field Hamiltonian can be diagonalized by means of a Bogoliubov transformation. We find that the Bogoliubov dispersion relations are given by (see Appendix A for derivations)

$$
\hbar \omega_{\phi, \mathbf{k}}^{ \pm}=\frac{1}{2}\left(\xi_{\phi, \mathbf{k}}-\xi_{\chi, \mathbf{k}}\right)+\Omega_{ \pm}
$$

and

$$
\hbar \omega_{\chi, \mathbf{k}}^{ \pm}=\frac{1}{2}\left(\xi_{\chi, \mathbf{k}}-\xi_{\phi, \mathbf{k}}\right)+\Omega_{ \pm}
$$

where $\xi_{\alpha, \mathbf{k}}=\epsilon_{\alpha, \mathbf{k}}-\mu_{\alpha}, \epsilon_{\alpha, \mathbf{k}}=\hbar^{2} k^{2} / 2 M_{\alpha}(\alpha=\phi, \chi)$, and

$$
\Omega_{ \pm}=\frac{1}{2} \sqrt{\frac{1}{2}|\Delta|^{2} \pm \frac{\left|\mathbf{F}_{\Delta}\right|}{2 f^{+}}+\left(\xi_{\phi, \mathbf{k}}+\xi_{\chi, \mathbf{k}}\right)^{2}}
$$

Here $|\Delta|^{2}=\sum_{m}\left|\Delta_{f, m}\right|^{2}$, the spin vector $\boldsymbol{F}_{\Delta}$ of the Cooper pairs is given by

$$
\boldsymbol{F}_{\Delta}=\boldsymbol{\Delta}^{\dagger} \cdot \boldsymbol{F} \cdot \boldsymbol{\Delta}=\sum_{m, m^{\prime}} \Delta_{f, m}^{*}(\mathbf{F})_{m, m^{\prime}} \Delta_{f, m^{\prime}}
$$

with $(\mathbf{F})_{m, m^{\prime}}$ being the spin- $f$ matrix, and $\Delta_{f, m}$ is given in Eq. (12).

In the absence of the spin degrees of freedom the Bogoliubov modes in Eqs. (14) and (15) describe quasiparticle excitations of fermions with mass and population imbalance [16, 17, 19]. To determine the spin structure we next derive and solve the gap equation for our system. After the Bogoliubov transformation the Hamiltonian is diagonalized as

$$
\begin{aligned}
\hat{H}= & \sum_{\sigma, \mathbf{k}} \hbar \omega_{\phi, \mathbf{k}}^{\sigma} \hat{\eta}_{\sigma, \mathbf{k}}^{\dagger} \hat{\eta}_{\sigma, \mathbf{k}}+\sum_{\sigma, \mathbf{k}} \hbar \omega_{\chi, \mathbf{k}}^{\sigma} \hat{\zeta}_{\sigma, \mathbf{k}}^{\dagger} \hat{\zeta}_{\sigma, \mathbf{k}} \\
& +\sum_{\mathbf{k}}\left(2 \xi_{\chi, \mathbf{k}}-\hbar \omega_{\chi, \mathbf{k}}^{+}-\hbar \omega_{\chi, \mathbf{k}}^{-}\right)-\sum_{m} \frac{\left|\Delta_{f, m}\right|^{2}}{2 g_{f}}
\end{aligned}
$$

where $\hat{\eta}_{\sigma, \mathbf{k}}$ and $\hat{\zeta}_{\sigma, \mathbf{k}}$ are the Bogoliubov-mode operators that reduce continuously to $\hat{\phi}_{\sigma, \mathbf{k}}$ and $\hat{\chi}_{\sigma, \mathbf{k}}$, respectively, as $|\Delta| \rightarrow 0$. There are additional contributions to the above Hamiltonian arising from the degenerate unpaired modes with ideal gas dispersion $\xi_{\phi, \mathbf{k}}=\hbar^{2} k^{2} / 2 M_{\phi}-\mu_{\phi}$; these terms, however, do not depend on the energy gap and are therefore not relevant for calculating the gap equation. The gap equation follows from the thermodynamic potential $\Omega$, which is determined from the partition function $Z=e^{-\beta \Omega}=\operatorname{Tr} e^{-\beta \hat{H}}$. Using the Hamiltonian in Eq. (18), we obtain

$$
\begin{aligned}
& \Omega=\sum_{\mathbf{k}}\left(2 \xi_{\chi, \mathbf{k}}-\hbar \omega_{\chi, \mathbf{k}}^{+}-\hbar \omega_{\chi, \mathbf{k}}^{-}\right)-\sum_{m} \frac{\left|\Delta_{f, m}\right|^{2}}{2 g_{f}} \\
& -\frac{1}{\beta} \sum_{\mathbf{k}, \alpha} \log \left(1+e^{-\beta \hbar \omega_{\alpha, \mathbf{k}}^{+}}\right)-\frac{1}{\beta} \sum_{\mathbf{k}, \alpha} \log \left(1+e^{-\beta \hbar \omega_{\alpha, \mathbf{k}}^{-}}\right) .
\end{aligned}
$$

The gap equations that follow from $d \Omega / d \Delta_{f, m}^{*}=0$ are given by

$$
\begin{aligned}
& -\frac{\Delta_{f, m}}{T_{f}^{2 B}}=\sum_{l= \pm 1} \sum_{\mathbf{k}}\left\{\frac{\Delta_{f, m}}{2} \frac{1}{\epsilon_{\phi, \mathbf{k}}+\epsilon_{\chi, \mathbf{k}}}\right. \\
& \left.+\frac{N\left(\hbar \omega_{\phi, \mathbf{k}}^{l}\right)+N\left(\hbar \omega_{\chi, \mathbf{k}}^{l}\right)-1}{2\left(\hbar \omega_{\phi, \mathbf{k}}^{l}+\hbar \omega_{\chi, \mathbf{k}}^{l}\right)}\left(\Delta_{f, m}+\frac{l}{2 f^{+}} \frac{d\left|\mathbf{F}_{\Delta}\right|}{d \Delta_{f, m}^{*}}\right)\right\} .
\end{aligned}
$$

Here by way of renormalization we have replaced the bare coupling constants $g_{f}$ with the two-body $T$-matrix $T_{f}^{2 B}$, where $T_{f}^{2 B}$ is related to $g_{f}$ by

$$
\frac{1}{T_{f}^{2 B}}=-\frac{1}{g_{f}}+\sum_{\mathbf{k}}\left(\epsilon_{\phi, \mathbf{k}}+\epsilon_{\chi, \mathbf{k}}\right)^{-1}
$$

From an experimental point of view, the two-body $T$ matrix is directly related to the $s$-wave scattering length $a_{f}$ with the total angular momentum $f$ of the two colliding atoms via $T_{f}^{2 B}=2 \pi a_{f} \hbar^{2} / M_{\mathrm{r}}$, where $M_{\mathrm{r}}=$ 
$M_{\phi} M_{\chi} /\left(M_{\phi}+M_{\chi}\right)$ is the reduced mass. If $\Delta_{f, m} \neq 0$ we can divide both sides of the gap equation by $\Delta_{f, m}$ and, as a result, the left-hand-side of the gap equation (20) becomes independent of $m$. To be consistent, the right-hand-side should also be independent of $m$, so $\frac{1}{\Delta_{f, m}} \frac{d\left|\mathbf{F}_{\Delta}\right|}{d \Delta_{f, m}^{*}}$ must be independent of $m$. This imposes a self-consistency relation for the order parameters and determines the possible superfluid phases. Before we investigate concrete examples, we will first investigate the connection of heteronuclear fermionic superfluids with spinor BECs in the next section.

\section{Ginzburg-Landau theory}

In this subsection we show that a heteronuclear fermionic superfluid with Cooper pairs having a nonzero spin can be maped onto a spinor BEC. For this purpose we formulate the Ginzburg-Landau theory for our system. We make use of the functional-integral formalism and as our starting point we take the action for the Hamiltonian $\hat{H}_{1 B}+\hat{V}$ discussed in Sec. 【A. The partition function $Z$ is expressed in terms of functional integrals over the fermionic fields $\phi_{\sigma}$ and $\chi_{\sigma}$ as

$$
Z=\int d[\phi] d\left[\phi^{*}\right] d[\chi] d\left[\chi^{*}\right] e^{-S / \hbar},
$$

where the action is given by

$$
\begin{aligned}
S\left[\phi^{*}, \phi, \chi^{*}, \chi\right] & =\int_{0}^{\hbar \beta} d \tau \int d \boldsymbol{x} \sum_{\sigma} \phi_{\sigma}^{*}(\boldsymbol{x}, \tau)\left(i \hbar \frac{\partial}{\partial \tau}-\frac{\hbar^{2} \nabla^{2}}{2 M_{\phi}}+V_{\phi, \sigma}^{\mathrm{ex}}(\boldsymbol{x})-\mu_{\phi}\right) \phi_{\sigma}(\boldsymbol{x}, \tau) \\
& +\int_{0}^{\hbar \beta} d \tau \int d \boldsymbol{x} \sum_{\sigma} \chi_{\sigma}^{*}(\boldsymbol{x}, \tau)\left(i \hbar \frac{\partial}{\partial \tau}-\frac{\hbar^{2} \nabla^{2}}{2 M_{\chi}}+V_{\chi, \sigma}^{\mathrm{ex}}(\boldsymbol{x})-\mu_{\chi}\right) \chi_{\sigma}(\boldsymbol{x}, \tau) \\
& +\frac{1}{2} \int_{0}^{\hbar \beta} d \tau \int d \boldsymbol{x} \sum_{m} g_{f} A_{f, m}^{*}(\boldsymbol{x}, \tau) A_{f, m}(\boldsymbol{x}, \tau) .
\end{aligned}
$$

The first two lines on the right-hand side describe free propagations of the fermion fields and the last line describes the two-body interaction which involves pairing amplitudes

$$
\begin{aligned}
& A_{f, m}(\boldsymbol{x}, \tau)= \\
& \sum_{\sigma, \sigma^{\prime}}\left\langle f, m \mid f_{\phi}, \sigma ; \frac{1}{2}, \sigma^{\prime}\right\rangle \phi_{\sigma}(\boldsymbol{x}, \tau) \chi_{\sigma^{\prime}}(\boldsymbol{x}, \tau) .
\end{aligned}
$$

We consider the homogeneous case here by setting the external potentials $V_{\chi, \sigma}^{\text {ex }}(\boldsymbol{x})$ and $V_{\phi, \sigma}^{\text {ex }}(\boldsymbol{x})$ to be zero. We decouple the interaction by means of a HubbardStratonovich transformation, i.e., we introduce a complex auxiliary field $\Delta_{f, m}(\boldsymbol{x}, \tau)$ that couples to the product of fields $\sum_{\sigma, \sigma^{\prime}}\left\langle f, m \mid f_{\phi}, \sigma ; \frac{1}{2}, \sigma^{\prime}\right\rangle \phi_{\sigma}(\boldsymbol{x}, \tau) \chi_{\sigma^{\prime}}(\boldsymbol{x}, \tau)$. The fourth-order term generated by the HubbardStratonovich transformation exactly cancels the interaction term in the action and the resulting action $S\left[\Delta^{*}, \Delta, \phi^{*}, \phi, \chi^{*}, \chi\right]$ depends only quadratically on the fermion fields $\phi_{\sigma}(\boldsymbol{x}, \tau)$ and $\chi_{\sigma}(\boldsymbol{x}, \tau)$ :

$$
\begin{aligned}
& S\left[\Delta^{*}, \Delta, \phi^{*}, \phi, \chi^{*}, \chi\right]=-\int_{0}^{\hbar} d \tau \int d \boldsymbol{x} \sum_{m} \frac{\left|\Delta_{f, m}\right|^{2}}{g_{f}} \\
& -\hbar \int_{0}^{\hbar} d \tau \int d \boldsymbol{x} \int_{0}^{\hbar} d \tau^{\prime} \int d \boldsymbol{x}^{\prime} \Psi^{*} \cdot \boldsymbol{G}^{-1} \cdot \boldsymbol{\Psi}
\end{aligned}
$$

where $\boldsymbol{\Psi}^{*}$ stands for a set of fields

$$
\Psi^{*}(\boldsymbol{x}, \tau)=\left(\phi_{f_{\phi}}^{*}(\boldsymbol{x}, \tau), \ldots \phi_{-f_{\phi}}^{*}(\boldsymbol{x}, \tau), \chi_{\frac{1}{2}}(\boldsymbol{x}, \tau), \chi_{-\frac{1}{2}}(\boldsymbol{x}, \tau)\right),
$$

and properties of the system are encapsulated in the Green's function matrix $\boldsymbol{G}$ which is expressed in terms of the noninteracting Green's function $\boldsymbol{G}_{0}$ and self-energy $\boldsymbol{\Sigma}$ as

$$
\boldsymbol{G}^{-1}\left(\boldsymbol{x}, \tau ; \boldsymbol{x}^{\prime}, \tau^{\prime}\right)=\boldsymbol{G}_{0}^{-1}\left(\boldsymbol{x}, \tau ; \boldsymbol{x}^{\prime}, \tau^{\prime}\right)-\boldsymbol{\Sigma} .
$$

The noninteracting Green's function $\mathbf{G}_{0}$ is the diagonal $\left(2 f_{\phi}+3\right) \times\left(2 f_{\phi}+3\right)$ matrix

$$
\begin{aligned}
& \mathbf{G}_{0}^{-1}\left(\boldsymbol{x}, \tau ; \boldsymbol{x}^{\prime}, \tau^{\prime}\right)= \\
& {\left[\begin{array}{cc}
G_{\phi, 0}^{-1}\left(\boldsymbol{x}, \tau ; \boldsymbol{x}^{\prime}, \tau^{\prime}\right) \mathbf{1} & 0 \\
0 & -G_{\chi, 0}^{-1}\left(\boldsymbol{x}^{\prime}, \tau^{\prime} ; \boldsymbol{x}, \tau\right) \mathbf{1}
\end{array}\right],}
\end{aligned}
$$


where $-G_{\alpha, 0}^{-1}\left(\boldsymbol{x}^{\prime}, \tau^{\prime} ; \boldsymbol{x}, \tau\right) \mathbf{1}(\alpha=\phi, \chi)$ is a $\left(2 f_{\phi}+1\right) \times$ $\left(2 f_{\phi}+1\right)$ matrix that satisfies

$$
\begin{aligned}
& G_{\alpha, 0}^{-1}\left(\boldsymbol{x}, \tau ; \boldsymbol{x}^{\prime}, \tau^{\prime}\right) \\
& =-\frac{1}{\hbar}\left\{\hbar \frac{\partial}{\partial \tau}-\frac{\hbar^{2} \boldsymbol{\nabla}^{2}}{2 M_{\alpha}}-\mu_{\alpha}\right\} \delta\left(\boldsymbol{x}-\boldsymbol{x}^{\prime}\right) \delta\left(\tau-\tau^{\prime}\right) .
\end{aligned}
$$

The self-energy $\boldsymbol{\Sigma}$ is a $\left(2 f_{\phi}+3\right) \times\left(2 f_{\phi}+3\right)$ matrix

$$
\hbar \Sigma=\left[\begin{array}{cc}
0 & V \\
V^{\dagger} & 0
\end{array}\right]
$$

where $V$ is the $\left(2 f_{\phi}+1\right) \times 2$ matrix given by $V_{\sigma, \sigma^{\prime}}=$ $\sum_{m}\left\langle f, m \mid f_{\phi}, \sigma ; 1 / 2, \sigma^{\prime}\right\rangle \Delta_{f, m}$. Since the action (25) depends only quadratically on the fermion fields, we can integrate them out and obtain an effective action

$$
\begin{aligned}
S^{\mathrm{eff}}\left[\Delta^{*}, \Delta\right]= & -\int_{0}^{\hbar \beta} d \tau \int d \boldsymbol{x} \sum_{m} \frac{\left|\Delta_{f, m}(\boldsymbol{x}, \tau)\right|^{2}}{g_{f}} \\
& -\hbar \operatorname{Tr}\left[\log \left(-\mathbf{G}^{-1}\right)\right] .
\end{aligned}
$$

The last term in Eq. (31) can be expanded in powers of $\Delta$ by using

$$
\mathbf{G}^{-1}=\mathbf{G}_{0}^{-1}-\mathbf{\Sigma}=\mathbf{G}_{0}^{-1}\left(1-\mathbf{G}_{0} \boldsymbol{\Sigma}\right),
$$

where the self-energy $\hbar \Sigma$ is given by Eq. (30), and therefore

$$
\begin{aligned}
-\hbar \operatorname{Tr}\left[\log \left(-\mathbf{G}^{-1}\right)\right]= & -\hbar \operatorname{Tr}\left[\log \left(-\mathbf{G}_{0}^{-1}\right)\right] \\
& +\hbar \sum_{m=1}^{\infty} \frac{1}{m} \operatorname{Tr}\left[\left(\mathbf{G}_{0} \mathbf{\Sigma}\right)^{m}\right] .
\end{aligned}
$$

The second-order $m=2$ term in the last term on the right-hand-side of Eq. (32) is given by

$$
\begin{aligned}
& \frac{\hbar}{2} \operatorname{Tr}\left[\left(\mathbf{G}_{0} \boldsymbol{\Sigma}\right)^{2}\right]=\frac{\hbar}{2} \int d \tau \int d \boldsymbol{x} \int d \tau^{\prime} \int d \boldsymbol{x}^{\prime} \int d \tau^{\prime \prime} \int d \boldsymbol{x}^{\prime \prime} \int d \tau^{\prime \prime \prime} \int d \boldsymbol{x}^{\prime \prime \prime} \\
& \operatorname{tr}\left[\mathbf{G}_{0}\left(\boldsymbol{x}, \tau ; \boldsymbol{x}^{\prime}, \tau^{\prime}\right) \boldsymbol{\Sigma}\left(\boldsymbol{x}^{\prime}, \tau^{\prime} ; \boldsymbol{x}^{\prime \prime}, \tau^{\prime \prime}\right) \mathbf{G}_{0}\left(\boldsymbol{x}^{\prime \prime}, \tau^{\prime \prime} ; \boldsymbol{x}^{\prime \prime \prime}, \tau^{\prime \prime \prime}\right) \boldsymbol{\Sigma}\left(\boldsymbol{x}^{\prime \prime \prime}, \tau^{\prime \prime \prime} ; \boldsymbol{x}, \tau\right)\right]
\end{aligned}
$$

where the trace operation $\operatorname{tr}[. .$.$] on the right-hand side$ means that we only take the sum of the diagonal elements of the $\left(2 f_{\phi}+3\right) \times\left(2 f_{\phi}+3\right)$ matrix $\mathbf{G}_{0} \boldsymbol{\Sigma} \mathbf{G}_{0} \boldsymbol{\Sigma}$. In the following we assume $\Delta_{f, m}$ to be independent of space and (imaginary) time. This allows us to separate the imaginary-time and space integrations from the matrix trace. For equal chemical potentials and equal masses we have $G_{\phi, 0}^{-1}\left(\boldsymbol{x}, \tau ; \boldsymbol{x}^{\prime}, \tau^{\prime}\right)=G_{\chi, 0}^{-1}\left(\boldsymbol{x}, \tau ; \boldsymbol{x}^{\prime}, \tau^{\prime}\right)$, and the integrals over the Green's functions reduce to those of the standard BCS theory.

The new contributions come from the matrix trace. For the second-order term we find after some straightforward algebraic manipulations that

$$
\operatorname{tr}\left[V^{\dagger} V\right]=\operatorname{tr}\left[V V^{\dagger}\right]=\sum_{m}\left|\Delta_{f, m}\right|^{2} .
$$

To evaluate the fourth-order term, we make use of

$$
\begin{aligned}
\operatorname{tr}\left[V^{\dagger} V V^{\dagger} V\right] & =\operatorname{tr}\left[V V^{\dagger} V V^{\dagger}\right] \\
& =\left(\operatorname{tr}\left[V^{\dagger} V\right]\right)^{2}-2 \operatorname{Det}\left[V^{\dagger} V\right] \\
& =\frac{1}{2}\left[\left(\sum_{m}\left|\Delta_{f, m}\right|^{2}\right)^{2}+\frac{\mathbf{F}_{\Delta} \cdot \mathbf{F}_{\Delta}}{f^{+2}}\right] .
\end{aligned}
$$

Combining these with conventional BCS theory we find that to the fourth order the thermodynamic potential, for equal masses and chemical potentials, is given by

$\Omega=\alpha_{f}(T) \sum_{m}\left|\Delta_{f, m}\right|^{2}+\beta\left(\left(\sum_{m}\left|\Delta_{f, m}\right|^{2}\right)^{2}+\frac{\left|\boldsymbol{F}_{\Delta}\right|^{2}}{f^{+^{2}}}\right)$,

where $\quad \alpha_{f}(T)=N(0) \log T_{\mathrm{c}}^{f} / T, \quad \beta=$ $7 \zeta(3) N(0) / 16\left(\pi k_{\mathrm{B}} T_{\mathrm{c}}^{f}\right)^{2}, \quad N(0)=2 M k_{\mathrm{F}} /(2 \pi \hbar)^{2}$ is the density of states at the Fermi energy, which is the same for both species since $M=M_{\phi}=M_{\chi}$ [44]. The 
critical temperature is given by

$$
T_{\mathrm{c}}^{f}=\frac{8 \epsilon_{\mathrm{F}}}{\pi k_{\mathrm{B}}} e^{\gamma-2} e^{-\pi / 2\left|k_{\mathrm{F}} a_{f}\right|} .
$$

The form of the thermodynamic potential in Eq. (36) is essentially the same as that of the homogeneous spinor BECs at zero magnetic field. Here, however, since $\beta>0$ the ground state is unmagnetized, i.e., $\boldsymbol{F}_{\Delta}=0$. Physically this result arises from the Pauli principle which forbids the fermionic constituents of the composite bosons to occupy the same state.

Moreover, we only find two kinds of fourth-order terms, i.e., the particle density and spin density, regardless of the value of the spin $f$. In contrast, for the case of spinor BECs there are $f+1$ different fourth-order terms. Therefore, all unmagnetized phases predicted in spin- $f$ BECs are degenerate in the present case.

\section{RESULTS}

As shown above, the heteronuclear fermionic superfluid has a Ginzburg-Landau free energy which is similar in form to that of a spinor BEC. We may thus expect similarities between the two systems insofar as mean-field theory is valid. To investigate this problem, we solve the gap equation (20) for the case of two spin- $1 / 2$ species whose total spin is either zero or one with the corresponding $s$-wave scattering length being $a_{0}$ or $a_{1}$. The phase which the gas condenses into is determined by the $s$-wave scattering length with the largest amplitude. For $f=0$ we immediately conclude that $\mathbf{F}_{\Delta}=0$. The Bogoliubov spectrum is the same as that of the conventional BCS theory. For total angular momentum $f=1$ we have

$$
\left|\mathbf{F}_{\Delta}\right|^{2}=\left(\sum_{m}\left|\Delta_{1, m}\right|^{2}\right)^{2}-3\left|\Theta_{1}\right|^{2},
$$

where the scalar quantity $\Theta_{1}=\left(-\Delta_{1,0}^{2}+\right.$ $\left.2 \Delta_{1,1} \Delta_{1,-1}\right) / \sqrt{3}$ is the same in form as the spinsinglet state pair amplitude of a spin-1 BEC 37]. If all $\Delta_{1, m}$ are nonzero, we find from the gap equation that either $\Theta_{1}=0$ or

$$
\frac{1}{\Delta_{1, m}} \frac{\partial \Theta_{1}}{\partial \Delta_{1, m}^{*}}=\frac{1}{\Delta_{1, m^{\prime}}} \frac{\partial \Theta_{1}}{\partial \Delta_{1, m^{\prime}}^{*}}
$$

holds for $m, m^{\prime}=0, \pm 1$. It follows from the latter condition that $\left|\Delta_{1,1}\right|=\left|\Delta_{1,-1}\right|$. Moreover, if we represent the order parameters as $\Delta_{1, m}=\left|\Delta_{1, m}\right| e^{i \theta_{1, m}}$, we find from the gap equation that $2 \theta_{1,0}=\theta_{1,1}+\theta_{1,-1}+\pi$. We also define the angle $\beta$ from the relation $\Delta_{1,0}=(|\Delta| / \sqrt{2}) \cos \beta$, where $|\Delta|^{2}=\sum_{m}\left|\Delta_{f, m}\right|^{2}$. Combining these, we find that

$$
\begin{aligned}
& \left(\Delta_{1,1}, \Delta_{1,0}, \Delta_{1,-1}\right)= \\
& |\Delta| e^{i \theta_{1,0}}\left(\frac{1}{\sqrt{2}} e^{i \alpha} \sin \beta, \cos \beta,-\frac{1}{\sqrt{2}} e^{-i \alpha} \sin \beta\right),
\end{aligned}
$$

where $\alpha=\theta_{1,1}-\theta_{1,0}$. This order parameter also describes the polar phase of the spin-1 Bose gas. From the condition $\Theta_{1}=0$, we obtain the phase relation $2 \theta_{1,0}=\theta_{1,1}+\theta_{1,-1}$ and the amplitude relation $\left|\Delta_{1,0}\right|^{2}=$ $2\left|\Delta_{1,1}\right|\left|\Delta_{1,-1}\right|$. From these results we find that the ferromagnetic order parameter is given by

$$
\begin{aligned}
& \left(\Delta_{1,1}, \Delta_{1,0}, \Delta_{1,-1}\right)= \\
& |\Delta| e^{i \theta_{1,0}}\left(e^{i \alpha} \cos ^{2} \frac{\beta}{2}, \sqrt{2} \sin \frac{\beta}{2} \cos \frac{\beta}{2}, e^{-i \alpha} \sin ^{2} \frac{\beta}{2}\right) .
\end{aligned}
$$

It can be shown that Eq. (41) is indeed a solution to the gap equation (20). Comparing the free energies of the polar and ferromagnetic phases we conclude that the ground state is polar. Because the order parameter of the polar phase has a $\boldsymbol{Z}_{2}$ symmetry, i.e., is invariant under the transformations $\alpha \rightarrow \alpha+\pi$ and $\beta \rightarrow \pi-\beta$, the meanfield theory predicts a quantum phase transition when we change the scattering lengths from $a_{1}>a_{0}$ to $a_{1}<a_{0}$, and vice versa.

To find solutions of the gap equation for higher values of the spin $f_{\phi}>1 / 2$ it is convenient to make use of the one-to-one correspondence between the fermionic heteronuclear gas and the spinor BEC derived in the previous section. The solutions for total spin 2 and spin 3 are of special interest because the correspond to the experimentally realized case of a mixture of ${ }^{171} \mathrm{Yb}$ (spin 1/2) and ${ }^{173} \mathrm{Yb}$ (spin 5/2). The spinor BECs with spin 2 and 3 have been described in the literature [24, 25, 26, 27]. and it is straightforward to verify that the possible groundstate solutions of the spin 2 and spin 3 BECs also satisfy the gap equation (20). For example, for the case of the spin 2 BEC cyclic and polar phases are possible [24, 25]. These phases can be distinguished from each other by the value of $\Theta_{2}=\left(\Delta_{2,0}^{2}-2 \Delta_{2,1} \Delta_{2,-1}+2 \Delta_{2,-2} \Delta_{2,2}\right) / \sqrt{5}$, which describes the formation of singlet pairs of spin-2 atoms. For spin 3 the solutions are more complex and have been discussed by Santos and Pfau [26] and Diener and Ho 27]. Also a complete classification of states and vortex excitations have been discussed in Refs. [28, 29].

\section{POSSIBLE EXPERIMENTAL REALIZATIONS}

The critical temperature needed to reach the weakcoupling limit is beyond experimental reach and in order to increase the critical temperature to experimentally accessible values we need to make use of optical Feshbach resonances [30] so that the effective interactions between the atoms are enhanced. Currently, optical Feshbach resonances for alkali atoms [32, 33] are not as effective as magnetic Feshbach resonances due to large atomic losses. It has been predicted [31, 34], however, that for groundstate alkaline-earth-metal atoms optical Feshbach resonances can be used to tune the scattering length over a wide range of values without suffering from the large 
atomic losses. As mentioned in the introduction, the fermionic isotopes of $\mathrm{Yb}$ have already been trapped optically [23], which makes them a prime candidate for realizing a heteronuclear fermionic superfluid with spin degrees of freedom. At present, experimental efforts are underway to find appropriate bound states in the long-lived ( $>10 \mathrm{sec}$ ) excited state potentials ${ }^{1} S_{0}+{ }^{3} P_{i=0.2}$ of Yb that can be used for optical Feshbach resonances [35]. If these efforts are successful we expect that the ground-states of the ${ }^{171} \mathrm{Yb}$ and ${ }^{173} \mathrm{Yb}$ mixture are the same as the unmagnetized phases of the spin 2 and 3 spinor BECs. Moreover, in the absence of symmetry breaking perturbations, the excitation spectrum for the unmagnetized groundstates is given by Eqs. (14) and (15) with $\left|\boldsymbol{F}_{\Delta}\right|=0$ and has the same form as the standard BCS theory. For a realistic gas, however, symmetry-breaking terms such as magnetic fields and dipole-dipole or intraspecies interactions are expected to lift the degeneracy and we plan to investigate this in future research.

\section{SUMMARY AND OUTLOOK}

In summary, we have presented a theory of superfluidity in heteronuclear Fermi-Fermi mixtures. We have shown that the heteronuclear fermionic superfluid has a Ginzburg-Landau free energy which is similar in form to that of a spinor BEC and, therefore, analogous mean-field ground states are expected for these two systems. This raises an important question concerning the nature of the many-body ground state in our case. For the bosonic case it has been shown that the exact many-body state is, in fact, not the polar state but a condensate of spin singlet pairs [38, 39, 40]. Explicitly, in the absence of a magnetic field the exact bosonic many-body state can be written as $|\psi\rangle \propto\left[\left(\left(\hat{a}_{0}^{\dagger}\right)^{2}-2 \hat{a}_{1}^{\dagger} \hat{a}_{-1}^{\dagger}\right) / \sqrt{3}\right]^{N / 2}|0\rangle$, where $a_{m}^{\dagger}$ creates a boson in hyperfine state $m=0, \pm 1$ with zero linear momentum and $N$ is the number of bosons. To perform a similar many-body analysis for the heteronuclear Fermi superfluids is beyond the scope of the present paper and will be left for future research. It is clear, however, that if the similarity at the mean-field level extends to the many-body case, the exact ground state is expected to be a condensate of spin-singlet states involving a quartet of fermions. Quartet superfluidity is of interest in strongly interacting quantum liquids [41, 42] and in the formation of two-pion states [43]. In these works, however, only single species gases were considered for which a fourfold degeneracy and moderate coupling are indispensible. Our work demonstrates the possibillity of creating a quartet condensate using only spin- $1 / 2$ particles because the Pauli principle does not prevent different species from occupying the same spin state. This unique feature of heteronuclear fermionic superfluidity merits further theoretical and experimental investigations.

\section{ACKNOWLEDGEMENTS}

This work was supported by a Grant-in-Aid for Scientific Research (Grant No. 17071005) and by a 21st Century COE program on "Nanometer-Scale Quantum Physics" from the Ministry of Education, Culture, Sports, Science, and Technology of Japan. D.D. acknowledges H. Takahashi and his group for stimulating discussions concerning the experimental implementations of this work and support by the Japan Society for the Promotion of Science (Project No. 18.06716).

\section{APPENDIX A: THE BOGOLIUBOV SPECTRUM OF A HETERONUCLEAR FERMIONIC SUPERFLUID}

Substituting the mean-field expression in Eq. (13) into Eq. (2), we obtain the following Hamiltonian for a homogeneous system:

$$
\begin{aligned}
\hat{H}= & \sum_{\mathbf{k}}\left(\begin{array}{c}
\phi_{f_{\phi}}^{\dagger} \\
\vdots \\
\phi_{-f_{\phi}}^{\dagger} \\
\chi_{1 / 2} \\
\chi_{-1 / 2}
\end{array}\right)\left(\begin{array}{cc}
\left(\xi_{\phi, \mathbf{k}}-\lambda\right) \mathbf{1} & V \\
V^{\dagger} & \left(-\xi_{\chi, \mathbf{k}}-\lambda\right) \mathbf{1}
\end{array}\right)\left(\begin{array}{c}
\phi_{f_{\phi}}^{*} \\
\vdots \\
\phi_{-f_{\phi}}^{*} \\
\chi_{1 / 2}^{\dagger} \\
\chi_{-1 / 2}^{\dagger}
\end{array}\right) \\
& +\sum_{\mathbf{k}} 2 \xi_{\chi, \mathbf{k}}-\sum_{m} \frac{\left|\Delta_{f, m}\right|}{g_{f}},
\end{aligned}
$$

where we define the $\left(2 f_{\phi}+1\right) \times 2$ matrix

$$
V=-\left(\begin{array}{cc}
V_{j, 1 / 2} & V_{j,-1 / 2} \\
V_{j-1,1 / 2} & V_{j-1,-1 / 2} \\
\vdots & \vdots \\
V_{-j, 1 / 2} & V_{-j,-1 / 2}
\end{array}\right)
$$

with matrix elements $V_{\sigma, \sigma^{\prime}}=$ $\sum_{m}\left\langle f, m \mid j, \sigma ; 1 / 2, \sigma^{\prime}\right\rangle \Delta_{f, m}$. The eigenvalue problem associated with this Hamiltonian can thus be written in a compact form as

$$
\left(\begin{array}{cc}
\left(\xi_{\mathbf{k}}-\lambda^{\prime}\right) \mathbf{1} & V \\
V^{\dagger} & \left(-\xi_{\mathbf{k}}-\lambda^{\prime}\right) \mathbf{1}
\end{array}\right)\left(\begin{array}{l}
u \\
v
\end{array}\right)=0 .
$$

Here, we have introduced the variable $\xi_{\mathbf{k}}=\left(\xi_{\phi, \mathbf{k}}+\xi_{\chi, \mathbf{k}}\right) / 2$ and the shifted eigenvalue $\lambda^{\prime}=\lambda-\left(\xi_{\phi, \mathbf{k}}-\xi_{\chi, \mathbf{k}}\right) / 2$. The vector $u$ has $2 j+1$ components and $v$ has two components. The highest order of $\lambda^{\prime}$ in the eigenvalue eqation is $\lambda^{\prime 2 j+3}$, which determines the maximum number of modes in the system. After working out the multiplications, we obtain the following coupled equations

$$
\left(\xi_{\mathbf{k}}-\lambda^{\prime}\right) \mathbf{1} u+V \cdot v=0
$$


and

$$
\left(-\xi_{\mathbf{k}}-\lambda^{\prime}\right) \mathbf{1} v+V^{\dagger} \cdot u=0
$$

We solve Eq. (A4) formally for $u$ and substitute the solution into Eq. A5 to obtain the following equation for $v$ :

$$
\left\{\left(-\xi_{\mathbf{k}}-\lambda^{\prime}\right) \mathbf{1}+V^{\dagger} \cdot \frac{1}{\xi_{\mathbf{k}}-\lambda^{\prime}} \mathbf{1} \cdot V\right\} v=0 .
$$

The eigenvalues of this equation can be obtained by solving the characteristic equation,

$$
\operatorname{Det}\left[\left(\xi_{\mathbf{k}}-\lambda^{\prime}\right)\left(-\xi_{\mathbf{k}}-\lambda^{\prime}\right) \mathbf{1}+V^{\dagger} V\right]=0 .
$$

Similarly, we can obtain for $u$ that

$$
\left\{\left(\xi_{\mathbf{k}}-\lambda^{\prime}\right) \mathbf{1}+V \cdot \frac{1}{-\xi_{\mathbf{k}}-\lambda^{\prime}} \mathbf{1} \cdot V^{\dagger}\right\} u=0 .
$$

The eigenvalues for this can be obtained by solving the characteristic equation

$$
\operatorname{Det}\left[\left(\xi_{\mathbf{k}}-\lambda^{\prime}\right)\left(-\xi_{\mathbf{k}}-\lambda^{\prime}\right) \mathbf{1}+V V^{\dagger}\right]=0 .
$$

While Eq. (A7) is a $2 \times 2$ eigenvalue equation, Eq. (A9) is a $\left(2 f_{\phi}+1\right) \times\left(2 f_{\phi}+1\right)$ eigenvalue equation which has $2 f_{\phi}-1$ degenerate solutions $\lambda= \pm \xi_{\mathbf{k}}$ in addition to the nontrivial solutions. The nontrivial solutions follow from Eq. (A7). We have,

$$
V^{\dagger} V=\left(\begin{array}{cc}
\sum_{j^{\prime}} V_{j^{\prime}, 1 / 2}^{*} V_{j^{\prime}, 1 / 2} & \sum_{j^{\prime}} V_{j^{\prime}, 1 / 2}^{*} V_{j^{\prime},-1 / 2} \\
\sum_{j^{\prime}} V_{j^{\prime},-1 / 2}^{*} V_{j^{\prime}, 1 / 2} & \sum_{j^{\prime}} V_{j^{\prime},-1 / 2}^{*} V_{j^{\prime},-1 / 2}
\end{array}\right)
$$

Using this, we obtain the eigenvalues $\lambda$ from Eq. (A7),

$$
\begin{aligned}
& \lambda=\frac{\xi_{\phi, \mathbf{k}}-\xi_{\chi, \mathbf{k}}}{2} \\
& \pm \sqrt{\left(\frac{\xi_{\mathbf{k}}}{2}\right)^{2}+\frac{1}{2} \operatorname{tr}\left[V^{\dagger} V\right] \pm \frac{1}{2} \sqrt{\left(\operatorname{tr}\left[V^{\dagger} V\right]\right)^{2}-4 \operatorname{Det}\left[V^{\dagger} V\right]}}
\end{aligned}
$$

Next, we express the trace and determinant terms appearing in the above eigenvalues in terms of physical quantities. Firstly, combining Eq. A10) with Eq. (A2), we obtain

$$
\operatorname{tr}\left[V^{\dagger} V\right]=\sum_{j^{\prime}, \sigma^{\prime}}\left|V_{j^{\prime}, \sigma^{\prime}}\right|^{2}=\sum_{m}\left|\Delta_{f, m}\right|^{2}
$$

The other term is given by

$$
\left(\operatorname{tr}\left[V^{\dagger} V\right]\right)^{2}-4 \operatorname{Det}\left[V^{\dagger} V\right]=\frac{\left|\boldsymbol{F}_{\Delta}\right|^{2}}{f^{+^{2}}},
$$

where $\boldsymbol{F}_{\Delta}$ is given by Eq. (17).
[1] M. Greiner, C. A. Regal, D. S. Jin, Nature 426, 537 (2003).

[2] M. W. Zwierlein, C. A. Stan, C. H. Schunck, S. M. F. Raupach, S. Gupta, Z. Hadzibabic, and W. Ketterle, Phys. Rev. Lett. 91, 250401 (2003).

[3] S. Jochim, M. Bartenstein, A. Altmeyer, G. Hendl, S. Riedl, C. Chin, J. H. Denschlag, and R. Grimm, Science 302, 2101 (2003).

[4] C. A. Regal, C. Ticknor, J. L. Bohn, and D. S. Jin, Nature 424, 47 (2003).

[5] M. Bartenstein, A. Altmeyer, S. Riedl, S. Jochim, C. Chin, J. H. Denschlag, and R. Grimm, Phys. Rev. Lett. 92, 120401 (2004).

[6] T. Bourdel, L. Khaykovich, J. Cubizolles, J. Zhang, F. Chevy, M. Teichmann, L. Tarruell, S. J. J. M. F. Kokkelmans, and C. Salomon, Phys. Rev. Lett. 93, 050401 (2004).

[7] G. B. Partridge, W. Li, R. I. Kamar, Y. an Liao, and R. G. Hulet, Science 311, 503 (2006).

[8] M. W. Zwierlein, C. H. Schunck, A. Shirotzek, and W. Ketterle, Nature 442, 54 (2006).

[9] M. W. Zwierlein, A. Shirotzek, C. H. Schunck, and W. Ketterle, Science 311, 492 (2006).

[10] A. G. Truscott, K. E. Strecker, W. I. McAlexander, G. B. Partridge, and R. G. Hulet, Science 291, 2570 (2001).
[11] F. Schreck, L. Khaykovich, K. L. Corwin, G. Ferrari, T. Bourdel, J. Cubizolles, and C. Salomon, Phys. Rev. Lett. 87, 080403 (2001).

[12] Z. Hadzibabic, C. A. Stan, K. Dieckmann, S. Gupta, M. W. Zwierlein, A. Görlitz, and W. Ketterle, Phys. Rev. Lett. 88, 160401 (2002).

[13] G. Roati, F. Riboli, G. Modugno, and M. Inguscio, Phys. Rev. Lett. 89, 150403 (2002).

[14] S. Ospelkaus, C. Ospelkaus, L. Humbert, K. Sengstock, and K. Bongs, Phys. Rev. Lett. 97, 120403 (2006).

[15] E. Wille, F.M. Spiegelhalder, G. Kerner, D. Naik, A. Trenkwalder, G. Hendl, F. Schreck, R. Grimm, T.G. Tiecke, J.T.M. Walraven, S.J.J.M.F. Kokkelmans, E. Tiesinga, and P.S. Julienne, arXiv 0711.2916 (2007).

[16] P. F. Bedaque, H. Caldas, and G. Rupak, Phys. Rev. Lett. 91, 247002 (2003).

[17] W. V. Liu and F. Wilczek, Phys. Rev. Lett. 90, 047002 (2003).

[18] M. Iskin and C. A. R. Sa de Melo, Phys. Rev. Lett. 97, 100404 (2006).

[19] G.-D. Lin, W. Yi, and L.-M. Duan, Phys. Rev. A 74, 031604(R) (2006).

[20] M. Taglieber, A.-C. Voigt, T. Aoki, T.W. Hänsch,and K. Dieckmann, arXiv 0710.2779 (2007).

[21] T.-L. Ho and S. Yip, Phys. Rev. Lett. 82, 247 (1999). 
[22] C. Honerkamp and W. Hofstetter, Phys. Rev. Lett. 92, 170403 (2004).

[23] T. Fukuhara, Y. Takasu, M. Kumakura, and Y. Takahashi, Phys. Rev. Lett. 98, 030401 (2007); K. Honda, Y. Takasu, T. Kuwamoto, M. Kumakura, Y. Takahashi, and T. Yabuzaki, Phys. Rev. A 66, 021401(R) (2002).

[24] C. V. Ciobanu, S. -K. Yip, and T.-L. Ho, Phys. Rev. A 61, 33607 (2000).

[25] M. Ueda and M. Koashi, Phys. Rev. A 65, 63602 (2002).

[26] L. Santos and T. Pfau, Phys. Rev. Lett. 96, 190404 (2006).

[27] R. B. Diener and T.-L Ho, Phys. Rev. Lett. 96, 190405 (2006).

[28] S. -K. Yip, Phys. Rev. A 75, 023625 (2007).

[29] R. Barnett, A. Turner, and E. Demler, Phys. Rev. Lett. 97, 180412, (2006).

[30] P. O. Fedichev, Y. Kagan, G. V. Shlyapnikov, and J. T. M. Walraven, Phys. Rev. Lett. 77, 2913 (1996).

[31] R. Ciurylo, E. Tiesinga, S. Kotochigova, and P. S. Julienne, Phys. Rev. A 70, 062710 (2004).

[32] F. K. Fatemi, K. M. Jones, and P. D. Lett, Phys. Rev. Lett. 85, 4462 (2000).
[33] M. Theis, G. Thalhammer, K. Winkler, M. Hellwig, G. Ruff, R. Grimm, and J. H. Denschlag, Phys. Rev. Lett. 93, 123001 (2004).

[34] R. Ciurylo, E. Tiesinga, and P. S. Julienne, Phys. Rev. A 71, 030701(R) (2005).

[35] Private communication.

[36] T. -L. Ho, Phys. Rev. Lett. 81, 742 (1998).

[37] M. Ueda, Phys. Rev. A, 63, 013601 (2000).

[38] C. K. Law, H. Pu, and N. P. Bigelow, Phys. Rev. Lett. 81, 5257 (1998).

[39] M. Koashi and M. Ueda, Phys. Rev. Lett. 84, 1066 (2000).

[40] T.-L. Ho and S. K. Yip, Phys. Rev. Lett. 84, 4031 (2000).

[41] H. Kamei and K. Miyake, J. Phys. Soc. Jpn. 74, 1911 (2005).

[42] G. Röpke, A. Schnell, and P. Schuck,P. Nozières, Phys. Rev. Lett. 80, 3177 (1998).

[43] T. Alm, G. Chanfray, P. Schuck, and G. Welke, Nucl. Phys. A612, 472 (1997).

[44] C. A. R. Sá de Melo, M. Randeria, and J. R. Engelbrecht, Phys. Rev. Lett. 71, 3202 (1993). 